\title{
Analysis Specified in Statistical Analysis Plan
}

National Cancer Institute

\section{Source}

National Cancer Institute. Analysis Specified in Statistical Analysis Plan. NCI Thesaurus.

Code C117753.

The analysis has been performed based on the statistical analysis plan. 